\title{
Mapping the submillimeter spiral wave in NGC 6946
}

\author{
P. B. Alton ${ }^{1}$, S. Bianchi ${ }^{2}$, J. Richer ${ }^{3}$, D. Pierce-Price ${ }^{3}$, and F. Combes ${ }^{4}$ \\ 1 Department of Physics \& Astronomy, University of Wales, PO Box 913, Cardiff CF2 3YB, UK \\ 2 ESO, Karl-Schwarzschild-Strasse 2, 85748 Garching bei Muenchen, Germany \\ 3 Cavendish Radio Laboratory, Madingley Road, Cambridge, UK \\ ${ }^{4}$ LERMA, Observatoire de Paris, 61 avenue de l'Observatoire, 75014 Paris, France
}

Received 19 March 2001 / Accepted 4 April 2002

\begin{abstract}
We have analysed SCUBA $850 \mu \mathrm{m}$ images of the (near) face-on spiral galaxy NGC 6946, paying particular attention to the subtraction of sky signal. A comparison with both $21 \mathrm{~cm} \mathrm{HI}$ and ${ }^{12} \mathrm{CO}(2-1)$ intensity maps reveals a tight correlation between dust thermal emission and molecular gas at the kiloparsec level. By means of a Monte Carlo radiative transfer model, we convert a $B-K$ colour image of NGC 6946 into a map of visual optical depth. The model yields maximum opacities since we assume that any increase in $B-K$ colour, with respect to the disk edge, is attributable solely to extinction by dust. The resultant map of visual optical depth relates well to the distribution of neutral gas $\left(\mathrm{HI}+\mathrm{H}_{2}\right)$ and implies a global gas-to-dust ratio of 90 (this value is a lower limit). There is no significant radial variation of this ratio: this can be understood, since the gas content is dominated by far by the molecular gas. The latter is estimated through the CO emission tracer, which is itself dependent on metallicity, similarly to dust emission. In the absence of a more objective tracer, it is not possible to derive the true gas-to-dust ratio. By comparing the radial profile of our visual optical depth map with that of the SCUBA image, we infer an emissivity (dust absorption coefficient) at $850 \mu \mathrm{m}$ that is 3 times lower than the value measured by COBE in the Milky Way, and 9 times lower than in NGC 891. We view this very much as a lower estimate, however, given our initial assumptions in deriving the visual opacity, and the possibility of underestimating the large-scale submm emission, the effect being more severe for the nearly face-on orientation of NGC 6946. A decomposition of the spiral structure half way out along the disk of NGC 6946 suggests an interarm optical depth of between 1 and 2 . These surprisingly high values represent $40-80 \%$ of the visual opacity that we measure for the arm region.
\end{abstract}

Key words. galaxies: general - galaxies: individual: NGC 6946 - galaxies: ISM - galaxies: photometry galaxies: spiral - radio continuum: galaxies

\section{Introduction}

Modern submillimeter (submm) and far-infrared (FIR) facilities such as the Submillimeter Common User Bolometer Array on JCMT (SCUBA), Institut de Radioastronomie millimétrique (IRAM) and the Infrared Space Observatory (ISO) have undertaken an invaluable rôle in re-evaluating the radial distributions and global masses of galactic dust in nearby spiral galaxies (e.g. Guélin et al. 1993; Guélin et al. 1995; Neininger et al. 1996; Alton et al. 1998a; Siebenmorgen et al. 1999). Nevertheless, detailed information about how grains are distributed with respect to the spiral arm structure, or how they relate at the kiloparsec level to the various gas phases $\left(\mathrm{HI}, \mathrm{H}_{2}\right.$ ) has been less forthcoming (Knapen \& Beckman 1996; Seigar \& James 1998; Rand et al. 1999;

Send offprint requests to: P. B. Alton,

e-mail: paul.alton@astro.cf.ac.uk
Alton et al. 2001). A major obstacle, in this respect, is the limited spatial resolution available with instruments such as ISO $\left(\sim 100^{\prime \prime}\right.$ at $\left.200 \mu \mathrm{m}\right)$ - barely sufficient to resolve the spiral arms in even the closest galaxies. At wavelengths closer to $1 \mathrm{~mm}$, ground-based arrays such as SCUBA and IRAM, afford considerably better spatial resolution $\left(\sim 10^{\prime \prime}\right)$ but emission arises from the RayleighJeans tail in this regime and, consequently, surface brightnesses are very low (usually $\sim 10 \mathrm{mJy} /$ beam). The sparse emission is particularly problematic for face-on spirals and it is precisely galaxies with this kind of inclination that we need to study if we are to understand how gas and dust relate to the spiral density wave.

At the global level several important trends have emerged from recent submm $/ \mathrm{mm}$ observations of spirals. For example, Dunne et al. (2000) have shown that the total $850 \mu \mathrm{m}$ flux density correlates more tightly with integrated $\mathrm{CO}(1-0)$ line emission than with the $21 \mathrm{~cm}$ 
HI line emission. The mid-IR emission ( 7 to 15 microns) detected with ISOCAM does not correlate well with HI and CO either (e.g. Genzel \& Cesarsky 2000; Frick et al. 2001). Total dust masses that include submm $/ \mathrm{mm}$ data now begin to show signs that they are comparable to dust masses inferred from optical extinction/reddening (Block et al. 1994; Alton et al. 1998b). Nevertheless, at the local level $(\sim 1 \mathrm{kpc})$ it is less clear how grain thermal emission relates to optical absorption lanes, star-forming regions, the diffuse interstellar medium (ISM) and, of course, the various gas phases $\left(\mathrm{HI}, \mathrm{H}_{2}, \mathrm{HII}\right)$.

In a recent paper, Alton et al. (2001) collated and examined the most up-to-date estimates of spiral disk opacity. What became obvious in this study is that relatively little is known about how dust is distributed with respect to the spiral arms and, in particular, whether interarm grain material is prevalent or not. The interarm region is particularly important because it occupies more than $50 \%$ of the disk and may therefore be crucial in determining the ability of spiral disks to block background light (Heisler \& Ostriker 1988; Fall \& Pei 1993; Gonzalez et al. 1998; Masci \& Webster 1999). Furthermore, the distribution of dust with respect to the spiral density wave may hold clues as to how gas is compressed along the spiral arms to form new stars. We should not forget that half of the metals in spiral galaxies are encapsulated in grains and consequently galactic dust provides a valuable "fossil record" of previous star-formation within the disk environment (Whittet 1992; Williams \& Taylor 1996; Edmunds \& Eales 1998).

Table 1. Basic properties of NGC 6946.

\begin{tabular}{llc}
\hline \hline Parameter & Value & Reference \\
\hline Type & Sc & de Vaucouleurs et al. (1991) \\
$R_{25}$ radius & $5.45^{\prime}$ & $"$ \\
RA (J2000) & $20^{\mathrm{h}} 34^{\mathrm{m}} 52^{\mathrm{s}} .3$ & $"$ \\
Dec (J2000) & $60^{\circ} 09^{\prime} 14.2^{\prime \prime}$ & $"$ \\
Distance & $10 \mathrm{Mpc}$ & Tacconi \& Young (1986) \\
Inclination & $30^{\circ}$ & Corradi \& Capaccioli (1991) \\
PA & $63^{\circ}$ & $"$ \\
\hline
\end{tabular}

In this paper, we address some of the issues outlined above by analysing data for NGC 6946. In most respects, this nearby $(10 \mathrm{Mpc})$ galaxy resembles a typical Sc spiral and is oriented favourably for a study of face-on spiral structure (Table 1). The centre of NGC 6946 evidently contains a large amount of recent star-formation (Tacconi \& Young 1990; Engargiola 1991). However, there is no evidence from optical and near-infrared (NIR) imaging of tidal disturbance and indeed, the disk appears quite isolated on the sky (except for the low surface brightness spiral Cep 1, at $0.4 \mathrm{Mpc}$ in projected distance, Burton et al. 1999). NGC 6946 is peculiar for its richness in CO emission, the derived molecular-to-atomic gas mass being quite high (Casoli et al. 1990). The density waves are not very regular nor contrasted, and in particular the total radio continuum emission is only slightly enhanced in spiral arms, indicating low compression (Boulanger \& Viallefond 1992). The contrast is even lower, when short spacings are added to interferometric maps (Frick et al. 2001). Also, polarized radio emission is concentrated in interarm regions (Beck \& Hoernes 1996).

The global FIR-to-blue luminosity ratio is close to unity which is characteristic of quiescent spirals rather than an object undergoing extensive starburst activity (Soifer et al. 1987; Bianchi et al. 2000a). Our submm data for NGC 6946 embrace a re-analysis of $850 \mu \mathrm{m}$ data collected by Bianchi et al. (2000b) as well as some additional mapping observations, at the same wavelength, accessed from the SCUBA archive. We begin by examining the relationship between submm emission and neutral gas (HI and $\mathrm{H}_{2}$; Sect. 3). A Monte Carlo radiative transfer model is then applied to a $B-K$ colour map of NGC 6946 with a view to comparing visual optical depth with dust thermal emission (Sect. 4). The latter process leads to a lower limit in the grain emissivity at $850 \mu \mathrm{m}$ (Sect. 5). Visual optical depth is then compared to column density of neutral gas in order to discover how the gas-to-dust ratio changes with galactocentric radius and gas-phase metallicity (Sect. 6). Finally, we examine the distributions of $\mathrm{HI}, \mathrm{H}_{2}$, submm thermal emission and visual optical depth with respect to the spiral density wave, providing estimates of opacity and gas column density for both arm and interarm regions in NGC 6946 (Sect. 7).

\section{Observational details}

Our submm data for NGC 6946 stem from two sources: 22 hours of exposure taken by ourselves during April and June 1998, and a further 5 hours of observations retrieved from the SCUBA archive. The former have been presented briefly in Bianchi et al. (2000b). All the data were reduced in a similar fashion to that described in Bianchi et al. but more attention was now given to the subtraction of the sky signal (which is strong with respect to the source signal). The Submillimeter Common User Bolometer Array (SCUBA) is mounted at the Nasmyth focus of the James Clerk Maxwell Telescope (JCMT) and provides simultaneous imaging at 450 and $850 \mu \mathrm{m}$ by virtue of a dichroic beamsplitter (Holland et al. 1999), with a field of view of $2.3^{\prime}$. The shortwave array $(450 \mu \mathrm{m})$ consists of 91 bolometers $\left(\mathrm{HPBW}=7.5^{\prime \prime}\right)$ whilst the longwave array $(850 \mu \mathrm{m})$ is composed of 37 elements $\left(\mathrm{HPBW}=14.7^{\prime \prime}\right)$. At the shorter wavelength, we detect little more than the NGC $6946 \mathrm{nu}-$ cleus and, therefore, our results will concentrate chiefly on the $850 \mu \mathrm{m}$ emission.

In scan-map mode, the telescope scans the source at a rate of 24 arcsec per second and at an angle allowing the source to be fully sampled (Pierce-Price et al. 2000). The scan length was $9^{\prime}$, and spacing $\sim 1^{\prime}$. During the scan, the secondary chops at a frequency of $7.8 \mathrm{~Hz}$ within the observed field facilitating the removal of sky background emission. Each scan-map is a convolution of the source 
with the chop and the profile of the source is eventually restored by a fourier transform (FT) technique. Scanmaps presented here cover most of the NGC 6946 disk $\left(D_{25} \simeq 10^{\prime}\right)$ and an individual scan took typically 6 min. In order to sample emission over various scale-sizes, we adopted chop throws of 20,30 and $65^{\prime \prime}$ along both the RA and Dec directions.

The dedicated SCUBA software package, SURF (Jenness 1997), was employed to clean, flatfield and calibrate the images as well as correct for atmospheric attenuation. Since the chop takes place within the source field, the software also uses the edges of each scan to remove a linear baseline corresponding to the sky. At $850 \mu \mathrm{m}$, the zenith optical depth was usually between 0.1 and 0.3 . Scan-maps of Uranus, processed in the same manner as the galaxy data, provided a calibration from Volts to Janskys for each night's observations. The uncertainty in this calibration is estimated to be $15 \%$.

The Emerson II technique enabled a final image to be produced from the chop-convolved data (Emerson 1995; Jenness et al. 1998; Holland et al. 1999). Scan-maps associated with each chop configuration were combined into a single image. Each of these 6 maps was then divided by the FT of the chop (a sine wave) in order to obtain the FT of the source. The FTs of the source were combined before the inverse FT process was carried out to produce the final image. Unfortunately, frequencies close to zero, corresponding to slow changes in the sky signal, are poorly sampled by this technique. This inadequacy is manifested by large-scale undulations in the convolved images for each chop configuration (Pierce-Price et al. 2000). The difficulty was alleviated to some extent by adopting an iterative approach in the data processing. The final image at $850 \mu \mathrm{m}$ was fed back into an earlier stage of the reduction in order to help separate sky fluctuations from object signal. In our case, this was repeated 4 or 5 times before no further change was noted in the final image. Even after this process, some fluctuations of scalesize $\sim 5^{\prime}$ were still apparent in the final image. Attempts to remove these fluctuations by fitting a polynomial surface or using an unsharp-mask technique were largely successful and gave very similar results. We estimate that the largest detectable angular scale is of order $5^{\prime}$. A diffuse emission with a flat radial distribution larger than $5^{\prime}$, the radius of NGC 6946, would not be detected. In the end, an unsharp-mask technique was selected. Here, the original image was smoothed with a Gaussian of size 5.5' $F W H M$ and this smoothed version subtracted from the original version in order to give the signal above the "local" zero point. The resultant image was adopted as our final image at $850 \mu \mathrm{m}$. The $1 \sigma$ random noise in this final image is estimated to be $4.2 \mathrm{mJy} / 16^{\prime \prime}$ beam. The additional uncertainty associated with the sky subtraction (in particular the frequencies close to zero) is believed to be between 1.0 and $1.5 \mathrm{mJy} / 16^{\prime \prime}$ beam. This systematic error was estimated by dividing the scan-maps up into 3 sets (each set taken on different dates), processing each data set separately and comparing the 3 resultant final images photometrically. Similarly, various trials in the skysubtraction process (polynomial surface, unsharp-masking etc.) suggest uncertainties of order $1 \mathrm{mJy} / 16^{\prime \prime}$ beam in establishing the zero baseline.

For comparison with the SCUBA data, a ${ }^{12} \mathrm{CO}(2-1)$ image of NGC 6946 was kindly made available to us by Sauty et al. (1998). These data were taken with the IRAM $30-\mathrm{m}$ telescope and have a spatial resolution of $13^{\prime \prime}$. A new $\mathrm{CO}(1-0)$ map made with the 30-m telescope with $22^{\prime \prime}$ resolution will be soon available (Walsh et al., in press) and is discussed in Frick et al. (2001). It is however very similar to that from Sauty et al. (1998). Nieten et al. (1999) detected $\mathrm{CO}(4-3)$, which indicates the presence of warm gas, as far as the outer spiral arm. Bianchi et al. (2000b) have already noted a strong correlation between the $850 \mu \mathrm{m}$ dust thermal emission and the ${ }^{12} \mathrm{CO}(2$ 1) emission line in NGC 6946 . However, the relationship is not completely linear and there is also evidence for a bifurcation in scatter-plots of two quantities (discussed below). A very extensive image of NGC $6946\left(26^{\prime} \times 26^{\prime}\right)$, taken in the $21 \mathrm{~cm}$ atomic hydrogen line, has been made available to us more recently by F. Boulanger. These data have a spatial resolution of $25^{\prime \prime}$ and combine both single-dish and interferometer data (Boulanger \& Viallefond 1992). Deep images in the $B$ and $K$ wavebands, taken with the KPNO 0.9-m and 2.3-m WIRO telescopes, were obtained in April 1996 and November 1995 respectively (Trewhella 1998). The $K$-band image was obtained by means of the $128 \times 128$ pixel Michigan Infrared Camera and covers an area of $7.4^{\prime} \times 7.4^{\prime}$.

\section{Relationship between submm, $\mathrm{CO}$ and $\mathrm{HI}$ emissions}

The submm emission is a function of dust column density and dust temperature, while the $\mathrm{HI}$ and $\mathrm{CO}$ emissions are function of gas column density and gas excitation. All three can be considered as gas tracers, with assumptions on gas-to-dust ratios, dust temperature and gas temperature and density.

The $850 \mu \mathrm{m}$ emission detected from NGC 6946 constitutes almost entirely thermal emission from dust grains (at $T \sim 15 \mathrm{~K}$ ) heated by stellar radiation (Israel et al. 1999). This assumption begins to break down for the central $60^{\prime \prime}$ of the disk where contamination by the ${ }^{12} \mathrm{CO}(3-2)$ line $(\lambda=870 \mu \mathrm{m})$ to the SCUBA longwave filter might be as high as 33\% (Mauersberger et al. 1999; Dumke et al. 2001). In Fig. 1, we show the SCUBA $850 \mu$ m image after smoothing to a spatial resolution of $18^{\prime \prime}(F W H M)$. The salient features of this image are similar to those described in detail by Bianchi et al. (2000b). The nuclear region is a strong source of submm radiation, with a maximum surface brightness of $250 \pm 40 \mathrm{mJy} / 18^{\prime \prime}$ beam at $850 \mu \mathrm{m}$. There are also local "hot-spots" situated at $\simeq 2.5^{\prime}$ away from the nucleus and position angles of $44^{\circ}, 99^{\circ}$ and $203^{\circ}$. These coincide with prominent HII regions in $B$-band and $\mathrm{H} \alpha$ images of NGC 6946 . The inner disk $\left(2.5^{\prime} \times 2.5^{\prime}\right)$ is reasonably well detected in our SCUBA image. 


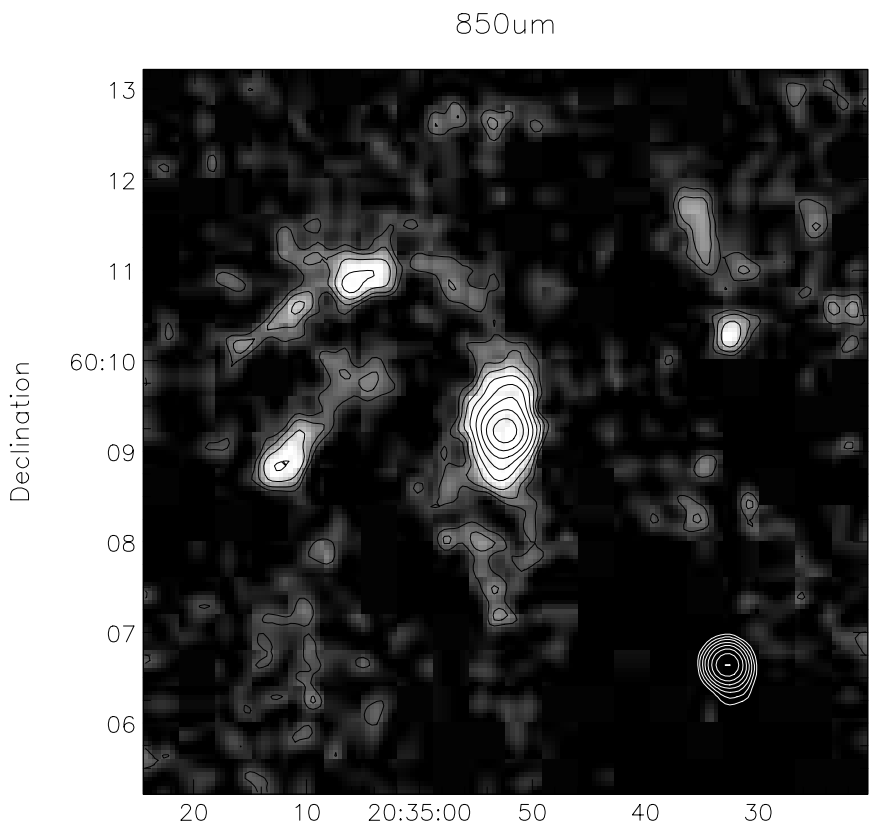

Right ascension

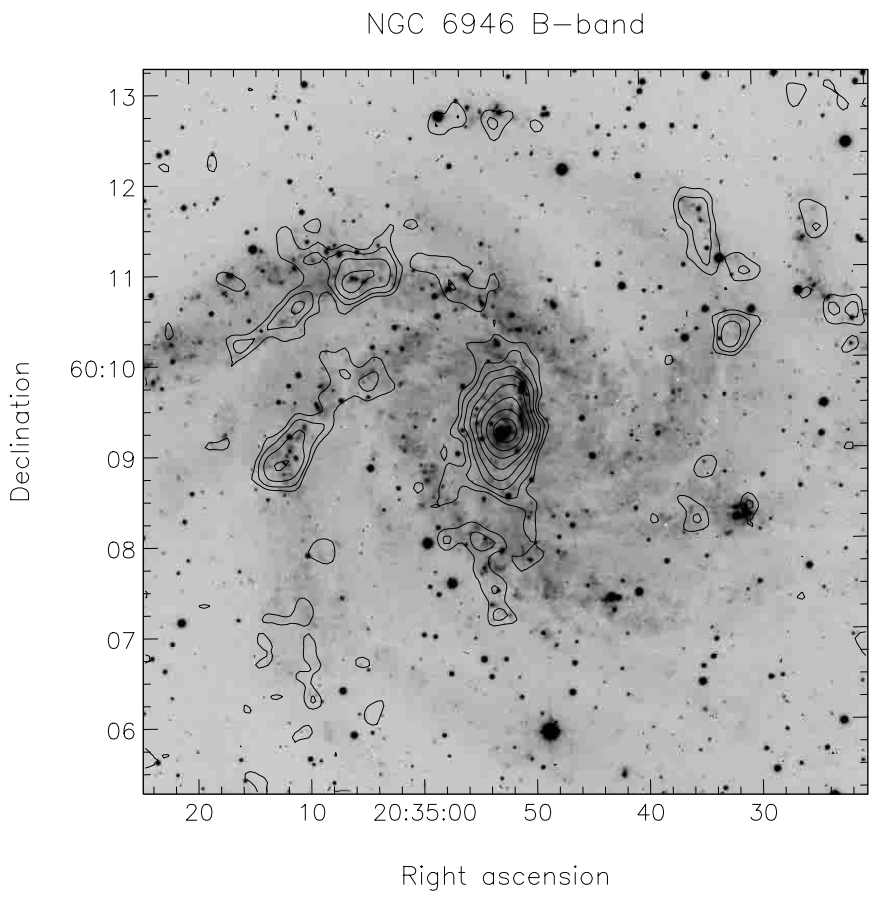

Fig. 1. SCUBA $850 \mu \mathrm{m}$ greyscale image (top) superimposed by $850 \mu \mathrm{m}$ contours. Isophotes start at $3 \sigma$ and are separated by $0.5 \mathrm{mag}$. The beam $\left(18^{\prime \prime} F W H M\right)$ is shown with white contours at the bottom-right of the plotted region. In the bottom figure, the submm isophotes have been projected onto a $B$-band image.

To compare, on equal terms, the distributions of neutral gas and dust thermal emission, we smoothed the ${ }^{12} \mathrm{CO}(2-1)$ and $850 \mu \mathrm{m}$ images to the same spatial resolution as the $21 \mathrm{~cm}$ HI map (25") using a circular Gaussian filter. Photometric sampling was then carried out, on all 3 images, for those regions where significant $850 \mu \mathrm{m}$ emission $(\geq 2 \sigma)$ had been detected. This was conducted using a $25^{\prime \prime}$ diameter aperture for a total of 46 regions. To convert the ${ }^{12} \mathrm{CO}(2-1)$ line emission to molecular hydrogen column density, $N\left(\mathrm{H}_{2}\right)$, we adopted 0.4 as the ratio of $\mathrm{CO}(2-1)$ to $\mathrm{CO}(1-0)$ intensity (as observed by Casoli et al. 1990 in the main disk). We then assumed $X=1.5 \times 10^{20} \mathrm{~cm}^{-2} \mathrm{~K} \mathrm{~km} \mathrm{~s}^{-1}$ for the conversion of $\mathrm{CO}(1-0)$ to $N\left(\mathrm{H}_{2}\right)$. We recognize sizeable uncertainties in our conversion parameters, in particular a factor 2 in the adopted " $X$ " factor. More insidious perhaps is the expected radial change in " $X$ " which is probably as large as any uncertainty in the global value (Neininger et al. 1996; Dumke et al. 1997). Furthermore, the ratio of $\mathrm{CO}(2-1)$ to $\mathrm{CO}(1-0)$ intensity, within the $25^{\prime \prime}$ of NGC 6946 , has been shown to be closer to unity rather than the value of 0.4 adopted here (Weliachew et al. 1988; Casoli et al. 1990). Fortunately, many of the inference we make in this paper are based on the main disk and exclude the central $1^{\prime}$ of the galaxy. It is this region which is likely to show dramatic changes in the conversion factors. Finally, we emphasize that we have assumed a fairly low value for $X$, consistent with recent gamma ray studies and the latest review articles of the $\mathrm{CO}(1-0)$ to $N\left(\mathrm{H}_{2}\right)$ conversion factor (Maloney 1990).

Figure 2 demonstrates how the submm emission relates to the hydrogen column density derived from $\mathrm{CO}$ and $21 \mathrm{~cm}$ HI maps. The top graph indicates a strong positive trend between molecular gas and $850 \mu \mathrm{m}$ surface brightness, $F_{850}$. Figure 2 also suggests little correspondance between atomic gas and the submm emission we detect. For the disk as a whole this is intuitively correct because the $850 \mu \mathrm{m}$ flux density is clearly concentrated towards the centre whereas NGC 6946, like so many other spirals, is known to possess a central depression in HI column density (Boulanger \& Viallefond 1992). Including the atomic gas in the total hydrogen column density does little to strengthen the correlation between neutral gas and $F_{850}$, primarily due to the dominance of $N\left(\mathrm{H}_{2}\right)$ at higher submm surface brightnesses. A least squares fit between total column of neutral hydrogen, $N\left(\mathrm{HI}+\mathrm{H}_{2}\right)$, and $F_{850}$ yields the following:

$$
\frac{N\left(\mathrm{HI}+\mathrm{H}_{2}\right)}{10^{21}}=(0.115 \pm 0.0052) \times F_{850}+(1.3 \pm 0.4)
$$

where $N\left(\mathrm{HI}+\mathrm{H}_{2}\right)$ and $F_{850}$ are expressed in atoms $/ \mathrm{cm}^{2}$ and $\mathrm{mJy} / 25^{\prime \prime}$ beam, respectively. The two fitted coefficients for the correlation with $N\left(\mathrm{H}_{2}\right)$ alone are $0.117 \pm$ 0.0057 for the slope and $-0.5 \pm 0.4$ for the zero point. The addition of HI slightly improves the correlation, but the effect is not very large, given the low HI column densities, with respect to the derived $\mathrm{H}_{2}$ ones. In order to derive a gas-to-dust ratio from this relationship we are first compelled to relate $F_{850}$ to the optical depth of dust in NGC 6946. This step is carried out in the next section. 

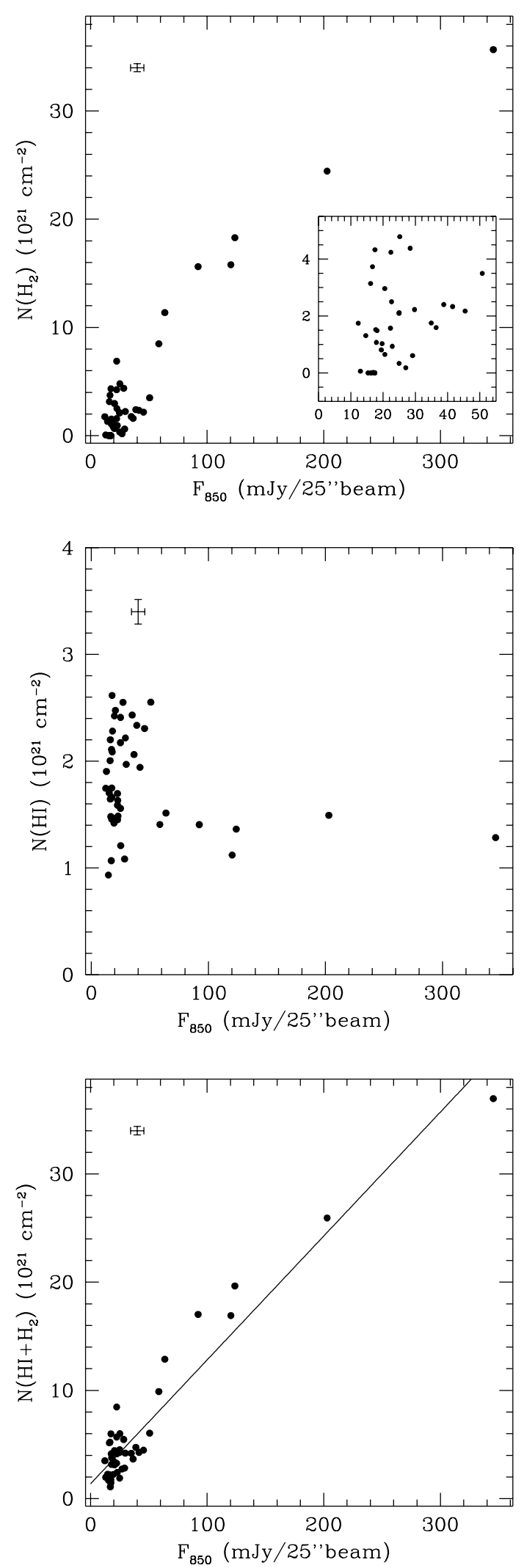

Fig. 2. Relationship between neutral gas and $850 \mu \mathrm{m}$ emission in NGC 6946 . The submm surface brightness is denoted by $F_{850}$ whilst $N(\mathrm{HI})$ and $N\left(\mathrm{H}_{2}\right)$ refer to the column density of atomic and molecular hydrogen respectively. In all cases, an error-bar at the top-left of the plotted region indicates the random error. The inset in the top graph magnifies data points occurring at lower $850 \mu \mathrm{m}$ surface brightness.

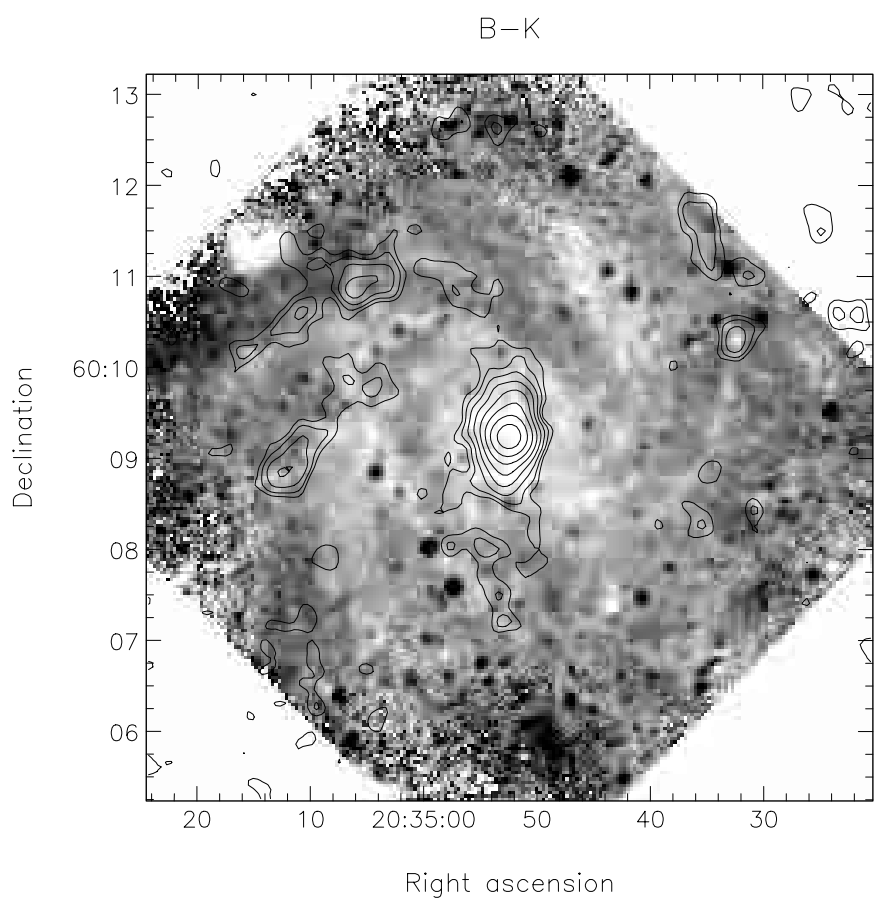

Fig. 3. $B-K$ colour image of NGC 6946 displayed in greyscale (reddened regions appear as white). This is quite similar to the extinction map of Trewhella (1998) combining optical and farinfrared. The $850 \mu \mathrm{m}$ contours appearing in Fig. 1 have been superimposed.

\section{Relationship between submm and reddening}

In this section, we relate thermal emission from dust at $850 \mu \mathrm{m}$ to reddening evident in optical images of NGC 6946 . The deep $B$ - and $K$-band images, alluded to in Sect. 2, were aligned and convolved to a common spatial resolution of $4.2^{\prime \prime}$ and the two images divided to produce a $B-K$ colour image in magnitudes. In Fig. 3 we show this reddening image with $850 \mu \mathrm{m}$ contours superimposed. NGC 6946 is typical of a "grand design" spiral galaxy with rather prominent spiral dust lanes (Trewhella 1998). We note the presence of extensive reddening $\sim 2^{\prime}$ north-west of the nucleus, while our $21 \mathrm{~cm} \mathrm{HI}$ and $\mathrm{CO}$ images give the impression of little atomic and molecular gas at this location. The filamentary structure in this part of the $B-K$ map is more suggestive of reddening by dust rather than an absence of blue stars.

Figure 3 reveals that the alignment between reddening lanes and dust thermal emission is surprisingly poor. We have already identified some of the submm emission as hot-spots situated close to optically-bright HII regions. Thus some of the brightest regions at $850 \mu$ m may well correspond to warm grains heated by young stars $(T=35 \mathrm{~K}$; Bianchi et al. 2000b). In contrast, optical extinction is not sensitive, in any direct sense, to grain temperature although dust clouds situated in front of bright, blue starforming regions will be detected quite readily in $B-K$ maps. The situation contrasts strongly with the conclusions drawn for the nearby spiral NGC 891 (Alton et al. 2000). Here, radial profiles along the major axis of this 
galaxy revealed a fairly good correspondance between optical reddening and $850 \mu \mathrm{m}$ emission. Furthermore, there were strong indications from the thermal spectrum of NGC 891 that the submm is dominated by cold grains (15$20 \mathrm{~K}$ ) rather than dust surrounding HII regions (Alton et al. 1998b). One possible explanation is that diffuse emission is dominating in NGC 6946, and remains undetected because of the face-on orientation (while it is detected in the edge-on NGC 891). Radial profiles of NGC 6946, to be discussed in Sect. 5, suggest a stronger link between visual optical depth and $850 \mu \mathrm{m}$ surface brightness than might be surmised from Fig. 3 .

In addition to accentuating the absorption lanes, our $B-K$ colour map highlights the presence of strong reddening towards the centre of NGC 6946. This is accompanied by an increase in $850 \mu \mathrm{m}$ surface brightness; both properties, taken together, suggest an increase in dust concentration towards the galaxy nucleus. For the analysis that follows, we assume that the reddening in NGC 6946 is solely attributable to extinction by dust (and not, for example, caused by a colour change in the underlying stellar population). We adopt the observed $B-K=3.1$ colour at a radius of $4.7^{\prime}\left(R \simeq 0.9 \times R_{25}\right)$ as the intrinsic, unreddened colour. This radius of $4.7^{\prime}$ is chosen because extinction is small there, and it is the largest radius where colours can be estimated properly. We then assume that any increase in the colour index with radius $(\Delta(B-K))$ arises from increasing optical depth. The results in radial variation (see Fig. 8) do not a posteriori show the need for a more sophisticated approach. A Monte Carlo radiative transfer model is then employed to infer the visual optical depth, $\tau_{\mathrm{V}}$, from $\Delta(B-K)$.

The assumption that we are measuring the true, underlying colour at a radius of $R=0.9 \times R_{25}$ is fairly robust - the optical depth is generally believed to be quite low near the $R_{25}\left(\tau_{\mathrm{V}} \simeq 0.2\right.$; Alton et al. 2001). However, our assumption that an increase in $\Delta(B-K)$ is purely attributable to dust is probably incorrect, although this viewpoint has been proposed by many previous authors (Peletier et al. 1995; Beckman et al. 1996). Peletier et al. (1995) find a statistical increase in the ratio of $B$-band radial scale-length to $K$-band radial scale-length for spiral disks more inclined to the line-of-sight. This effect is most readily explained in terms of dust reddening because the ratio would not change if the scale-lengths were determined by the stellar population gradient. In contrast, for a sample of 86 face-on spirals, de Jong (1996) concluded that the change in scale-length across several optical and NIR passbands could not be understood without gradients in star-formation and metallicity being considered as a major factor. The assumption in this paper that increased $B-K$ colour originates purely from increased optical depth will lead us to overestimate the opacity of the NGC 6946 disk. Furthermore, since the stellar population along the spiral arms is usually considered younger, and therefore bluer, than adjacent interarm regions, we will tend to both overestimate the amount of interarm dust and, therefore,

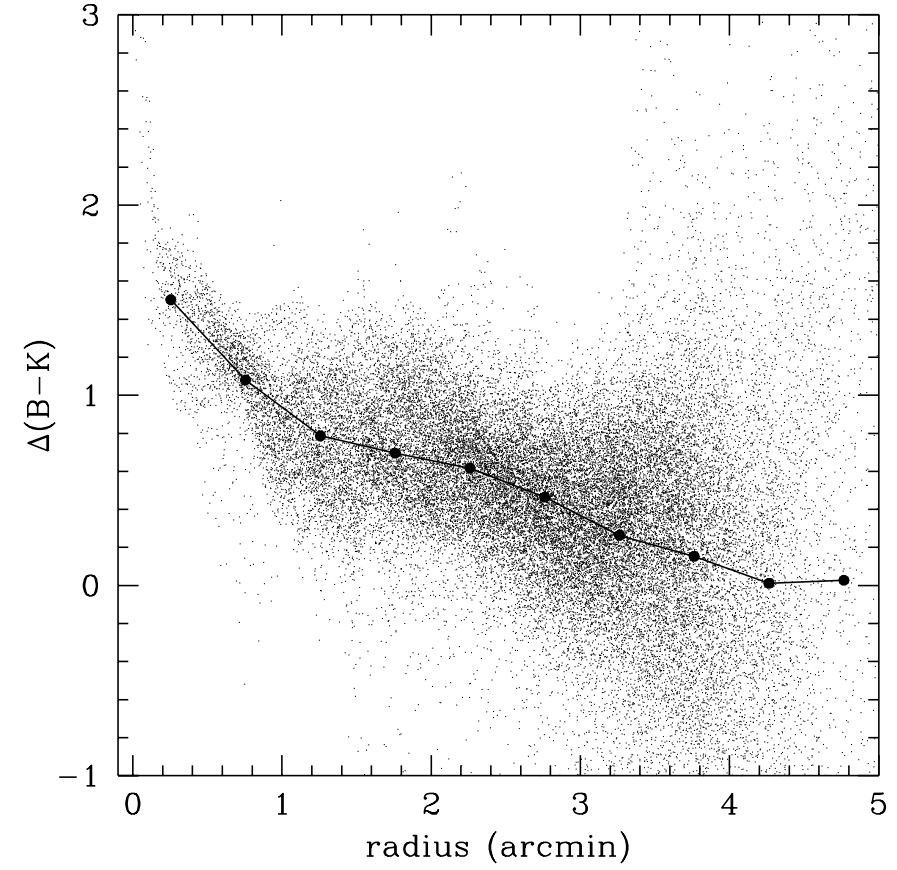

Fig. 4. A breakdown of the $B-K$ colour in Fig. 3. Dots represent the difference in colour of each pixel, $\Delta(B-K)$ with respect to the colour at the disk edge $\left(R=4.7^{\prime}\right)$. The baseline at $R=4.7^{\prime}$ has been established by means of an azimuthallyaveraged profile (solid circles and solid line).

overlook the propensity for dust to clump along the spiral arms (Knapen \& Beckman 1996).

Figure 4 provides a breakdown of the reddening measured in Fig. 3 on a pixel-by-pixel basis. The azimuthallyaveraged radial profile in $B-K$ colour has been superimposed. We interpret $\Delta(B-K)$ by means of the Monte Carlo radiative transfer (RT) model developed by Bianchi et al. (1996). This simulation assumes smooth distributions of both stars and dust which decline exponentially with both increasing galactocentric radius and height above the midplane. The light paths of optical and NIR photons are followed, through a succession of scattering interactions, until absorption takes place or the photon in question exits from the galaxy. By comparing the number of photons that escape from each part of the object with the quantity emitted by stars in the underlying disk, it is possible to relate observed colour to visual optical depth.

For NGC 6946, we adopt a "standard geometry" with dust grains extending to half the scale-height of the stellar disk and the dust radial scale-length fixed at 1.5 times the stellar scale-length. Acceptable variations in the scaleheight and scale-lengths of dust with respect to the stars (Trewhella 1997; Bianchi 1999) lead to uncertainties of $\simeq 33 \%$ in the implied visual optical depth over most of the disk ( $\sim 50 \%$ for the central arcminute). An $R^{1 / 4}$ bulge was added to the stellar light so that, in total, bulge stars contributed $\frac{1}{10}$ of the total optical+NIR luminosity of the galaxy. Finally, it should be noted that no account is taken of dust clumping in the model which normally would lead 


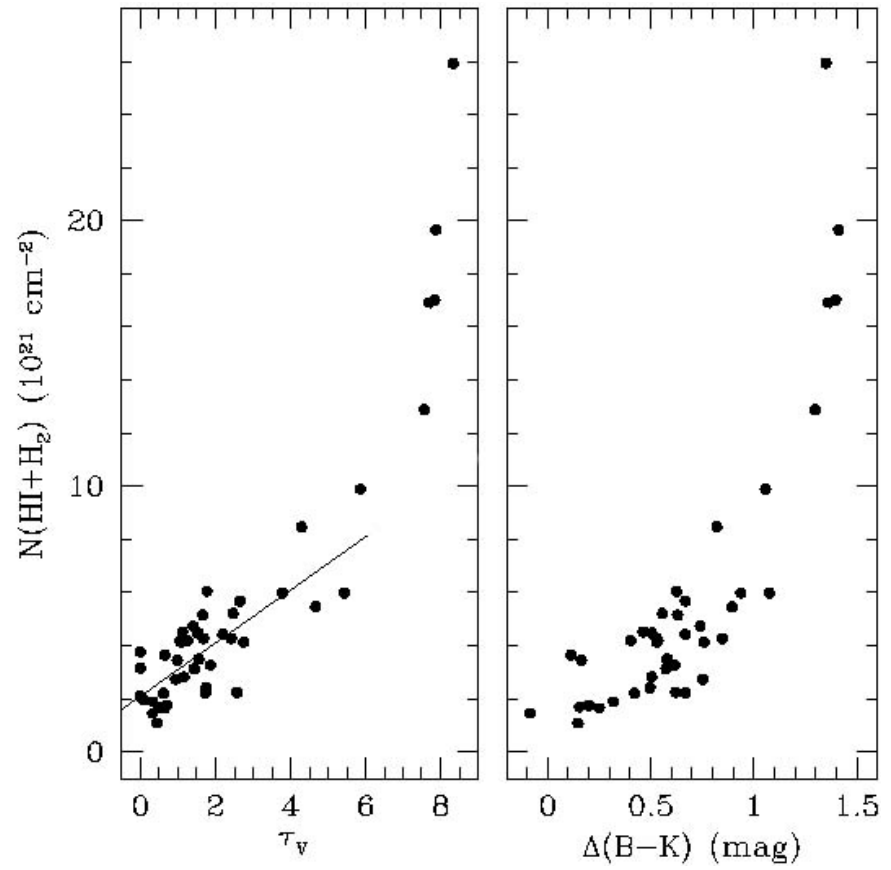

Fig. 5. Neutral hydrogen column density $\left(\mathrm{H}_{2}+\mathrm{HI}\right)$ plotted against both $B-K$ colour and visual optical depth, $\tau_{\mathrm{V}}$. The latter has been inferred from a Monte Carlo radiative transfer simulation. A least-squares linear fit between gas column density and $\tau_{\mathrm{V}}$ is represented by the solid line (excluding $\tau_{\mathrm{V}}>7$ ). The corresponding relationship in the Milky Way is also linear but with a gradient approximately twice that shown for NGC 6946.

us to underestimate the true optical depth by $50-100 \%$ (Witt \& Gordon 2000; Bianchi et al. 2000c).

Rather than attempt a global fit between the observed and simulated $B-K$ colours, each pixel in Fig. 3 was treated independently. A series of simulations were run, with the face-on, visual optical depth through the centre of the galaxy model varying between 2 and 10. Overall, the technique worked well; a notable exception being within $45^{\prime \prime}$ of the nucleus where the observed $\Delta(B-K)$ was too high to give a reliable indication of the underlying optical depth $\left(B-K\right.$ effectively saturates as $\tau_{\mathrm{V}}$ approaches $\left.\sim 10\right)$.

Using the method outlined above a map of visual optical depth was created to the same spatial resolution as our $K$-band image $\left(4.2^{\prime \prime} F W H M\right)$. To compare with the SCUBA image (next section), the $\tau_{\mathrm{V}}$ map was then smoothed to $18^{\prime \prime}$, yielding a central, face-on opacity of $\tau_{\mathrm{V}}^{0}=7.6$ and an exponential scale-length of $98^{\prime \prime}$, close to that inferred from ISO $200 \mu \mathrm{m}$ images. We conclude that the visual optical depths derived from our RT model are reasonable.

Figure 5 shows how the column density of neutral hydrogen in NGC 6946, $N\left(\mathrm{HI}+\mathrm{H}_{2}\right)$, relates to both reddening, $\Delta(B-K)$, and the optical depth derived from RT modelling, $\tau_{\mathrm{V}}$. If we exclude central regions saturated in
$B-K$ (and corresponding to $\tau_{\mathrm{V}}>6$ ), a strong linear relationship emerges as follows (correlation coefficient 0.91):

$\frac{N\left(\mathrm{HI}+\mathrm{H}_{2}\right)}{10^{21}}=(1.0 \pm 0.1) \tau_{\mathrm{V}}+(2.1 \pm 0.3)$.

This is half the corresponding value measured within the solar neighborhood $\left(N\left(\mathrm{HI}+\mathrm{H}_{2}\right)=2.1 \times 10^{21} \tau_{\mathrm{V}}\right.$; Bohlin et al. 1978), and could be due to the difference in metallicity. To obtain a total dust mass, we now convert $\tau_{\mathrm{V}}$ into dust column density and integrate over the whole disk. To do this, we employ the relation given by Xilouris et al. (1997) corresponding to "classical" Milky Way grains of size $0.1 \mu \mathrm{m}$ and material density $3 \mathrm{~g} \mathrm{~cm}^{-3}$ (Hildebrand 1983). Thus:

$M_{\mathrm{D}}=\int 0.27 \tau_{\mathrm{V}}^{0}(R) 2 \pi R \mathrm{~d} R$

where the integral is over radius $R$, in metres, along the disk. Here, $\tau_{\mathrm{V}}^{0}(R)$ is the face-on optical depth at $R$ and $M_{\mathrm{D}}$ is the total dust mass in g. Integrating Eq. (3) out to a radius $R \sim 4^{\prime}$, we derive $M_{\mathrm{D}}=8.6 \times 10^{7} M_{\odot}$ for NGC 6946. Integrating $N\left(\mathrm{HI}+\mathrm{H}_{2}\right)$ over the same region leads us to a global gas-to-dust ratio of 90 . This value is consistent with the somewhat shallower gradient derived in Eq. (2) compared with the solar neighborhood. Given our fundamental assumption in the derivation of $\tau_{\mathrm{V}}$ (that $B-K$ increases due to extinction only), our estimate of the gas-to-dust ratio must be considered a lower limit.

Let us note that this value of 90 for the gas-to-dust ratio is about a factor 2 lower than the average of 200 found towards 7 spiral galaxies (including NGC 6946) by Alton et al. (1998a). This is due essentially to the lower $X=\mathrm{H}_{2} / \mathrm{CO}$ conversion ratio adopted here $(X=1.5$ instead of $\left.2.8 \times 10^{20} \mathrm{~cm}^{-2}\left(\mathrm{~K} \mathrm{~km} \mathrm{~s}^{-1}\right)^{-1}\right)$. Also the $850 \mu \mathrm{m}$ SCUBA emission could trace even colder dust than the ISO $200 \mu \mathrm{m}$ emission used by Alton et al. (1998a). For comparison, the gas-to-dust ratio for the solar neighborhood is 150 , and 100-300 for NGC 891, according to the adopted conversion ratio or dust emissivity (Guélin et al. 1993; Alton et al. 2000).

\section{Inferred submm emissivity}

The submm emission is not only a function of the dust column density and the dust temperature, but also of the nature of grains, which is reflected in their emissivity $Q$ at various wavelengths.

A comparison between the SCUBA image and the optical depth just derived from RT modelling should allow us to constrain the $850 \mu \mathrm{m}$ emissivity of dust grains in NGC 6946, $Q(850 \mu \mathrm{m})$. The mass absorption coefficient $(\kappa)$, which is often used, is related to the grain emissivity as follows: $\kappa_{850 \mu \mathrm{m}}=\frac{3 Q(850 \mu \mathrm{m})}{4 a \rho}$, where $a$ and $\rho$ are the radius and material density of the grains respectively (Hildebrand 1983). It can be shown (e.g. Alton et al. 2000) that optical depth and submm brightness are related as follows:

$\tau_{\mathrm{V}}^{0}=\frac{Q(V)}{Q(850 \mu \mathrm{m})} \frac{1.67 \times 10^{-18}}{B(850 \mu \mathrm{m}, T)} \quad \cos (i) \quad F_{850}$ 


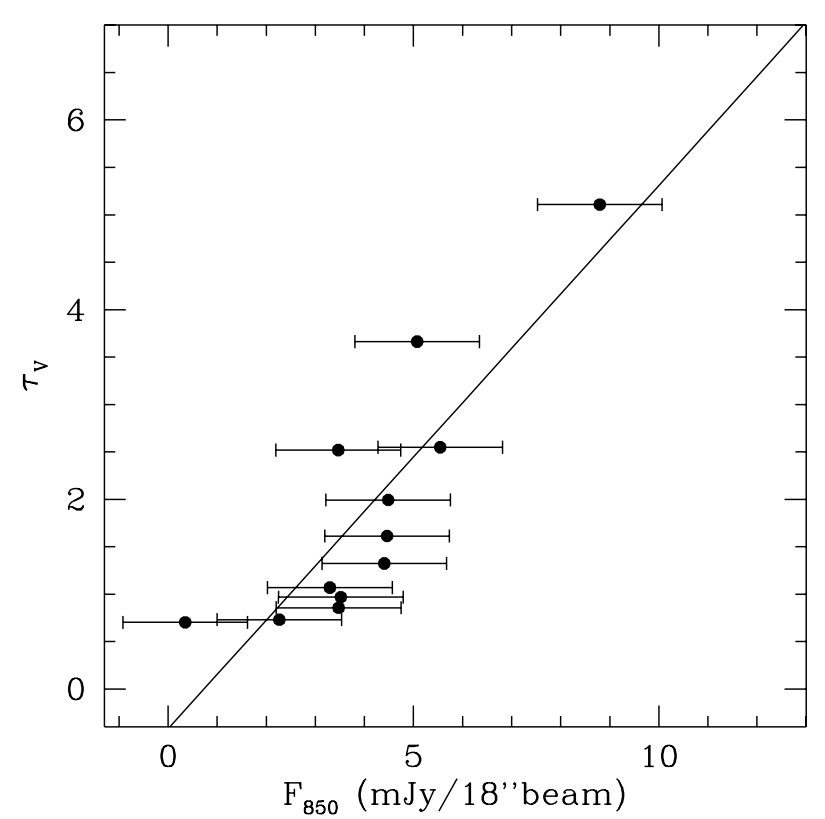

Fig. 6. The relationship between visual optical depth $\left(\tau_{\mathrm{V}}\right)$ and $850 \mu \mathrm{m}$ surface brightness $\left(F_{850}\right)$ for NGC 6946 . Errorbars are only shown for $F_{850}$ as the values for $\tau_{\mathrm{V}}$ are almost certainly upper limits. The solid line shows a least-squares linear fit to the data (see text).

where $\tau_{\mathrm{V}}^{0}$ is the face-on optical depth, $Q(V)$ is the emissivity at $\lambda=0.55 \mu \mathrm{m}, i$ is the inclination of the disk $\left(i=90^{\circ}\right.$ for edge-on), $F_{850}$ denotes the surface brightness of the $850 \mu \mathrm{m}$ map, in $\mathrm{Jy} / 18^{\prime \prime}$ beam, and $B(850 \mu \mathrm{m}, T)$ refers to the blackbody intensity at $850 \mu \mathrm{m}$ for grains of temperature $T$. We can see that an estimate of $\frac{\tau_{\mathrm{V}}}{F_{850}}$ would serve as a handle on $\frac{Q(V)}{Q(850 \mu \mathrm{m})}$.

With current observational limits there exists almost an order of magnitude uncertainty in $Q(850 \mu \mathrm{m})$ (and hence $\frac{Q(V)}{Q(850 \mu \mathrm{m})}$, since $Q(V)$ is relatively well constrained to $\sim 1.5$; Whittet 1992). Indeed, emissivity values for wavelengths close to $1 \mathrm{~mm}$ are often extrapolated from observations of Galactic reflection nebulae near 100-200 $\mu \mathrm{m}$ (see Alton et al. 2000 for a full discussion). At the same time we cannot overstate the importance of determining the submm/mm emissivity because, once $Q(\lambda)$ is known, submm/mm observations can then be furnished with visual optical depths in a relatively straightforward manner (Hildebrand 1983; Hughes et al. 1993).

Radial profiles were fitted to both SCUBA and visual optical depth maps (both after smoothing to a spatial resolution of $\left.18^{\prime \prime}\right)$. Values pertaining to the central arcminute of the object were discarded due to suspected saturation in the $B-K$ colour. Figure 6 shows that optical depth $\tau_{\mathrm{V}}$ compares rather well to submm surface brightness $F_{850}$, when azimuthally averaged (while it was not true locally, cf. Fig. 3). A least-squares best fit between optical depth and submm brightness yielded the following:

$\frac{\tau_{\mathrm{V}}}{F_{850}}=573 \pm 108$
Given the already mentioned uncertainties on $\tau_{\mathrm{V}}$ and $F_{850}$, this value of the ratio is an upper limit. If we are to derive $\frac{Q(V)}{Q(850 \mu \mathrm{m})}$ from Eqs. (4) and (5) we must have some notion of the grain temperature $T$. Unfortunately, no signal was recorded from the main disk of NGC 6946 during our $450 \mu \mathrm{m}$ SCUBA observations (which would have allowed us to plot the thermal spectrum). ISO $200 \mu \mathrm{m}$ images of NGC 6946, in conjunction with IRAS $100 \mu \mathrm{m}$ HiRes data, suggest dust temperatures close to $20 \mathrm{~K}$ in the main disk. RT modelling by Bianchi et al. (2000a) points to $T$ falling from $23 \mathrm{~K}$ at the centre of NGC 6946 to $17 \mathrm{~K}$ at the $R_{25}$ radius (similar to values proposed for the diffuse Galactic ISM; Mathis et al. 1983; Draine \& Lee 1984; Reach et al. 1995; Masi et al. 1995). Observations at both 450 and $850 \mu \mathrm{m}$ for the nearby spirals NGC 891 and NGC 7331 suggest that $90 \%$ of galactic dust maintains a temperature between 15 and $20 \mathrm{~K}$ (Alton et al. 1998b; Alton et al. 2001; see also Guélin et al. 1993). To translate $\frac{\tau_{\mathrm{V}}}{F_{850}}$ into $\frac{Q(V)}{Q(850 \mu \mathrm{m})}$ we adopt $T=18 \mathrm{~K}$. We note that a $50 \%$ uncertainty in $T$ will produce a $50 \%$ variation in $\frac{Q(V)}{Q(850 \mu \mathrm{m})}$ since $F_{850} \propto T$ in the Rayleigh-Jeans tail of the spectrum. The substitution of temperature into Eq. (4) yields $\frac{Q(V)}{Q(850 \mu \mathrm{m})} \simeq 1.4 \times 10^{5}$ and a mass absorption coefficient of $\kappa(850 \mu \mathrm{m}) \simeq 0.26 \mathrm{~cm}^{2} \mathrm{~g}^{-1}$ is we assume $Q(V)=1.5$ and grains of radius $0.1 \mu \mathrm{m}$ and material density $3 \mathrm{~g} \mathrm{~cm}^{-3}$ (see Fig. 7).

The implied $850 \mu \mathrm{m}$ emissivity is a factor 3 lower than that measured by COBE for diffuse Milky Way dust (suggesting we detect too little $850 \mu \mathrm{m}$ radiation compared with the optical depth inferred from RT modelling). However, we recall that our $\tau_{\mathrm{V}}$ values are only upper limits as we assumed from the outset that $B-K$ only changes due to extinction. The aforementioned $850 \mu \mathrm{m}$ emissivity should therefore be considered a lower limit. Also, there might be an underestimation of the emissivity, due to the possible existence of spatially flat diffuse emission and the uncertainty in the background subtraction. In NGC 891, the submm emissivity was estimated $9 \times$ higher than that for NGC 6946 (Alton et al. 2000), which might be due to more thorough detection of the diffuse emission, because of edge-on orientation.

\section{Gas-to-dust ratio}

Figure 8 reveals how the derived gas content varies with respect to dust, with distance from the nucleus.

In one case, the optical depth derived from RT modelling has been used to infer the dust mass per unit area (via Eq. (3)). The gas surface density includes both atomic and molecular gas (the latter based on $X=$ $1.5 \times 10^{20} \mathrm{~cm}^{-2} \mathrm{~K} \mathrm{~km} \mathrm{~s}^{-1}$ ). The radial gas-to-dust ratio has also been calculated using the submm profile discussed in Sect. 5 and converting $F_{850}$ to $\tau_{\mathrm{V}}$ with Eq. (5). The normalisation of $F_{850}$ to $\tau_{\mathrm{V}}$ explains why the mean gas-to-dust ratio indicated by both gas-to-dust profiles in Fig. 8 is more or less the same. Again, it must be emphasized here that both curves are only lower limits. 


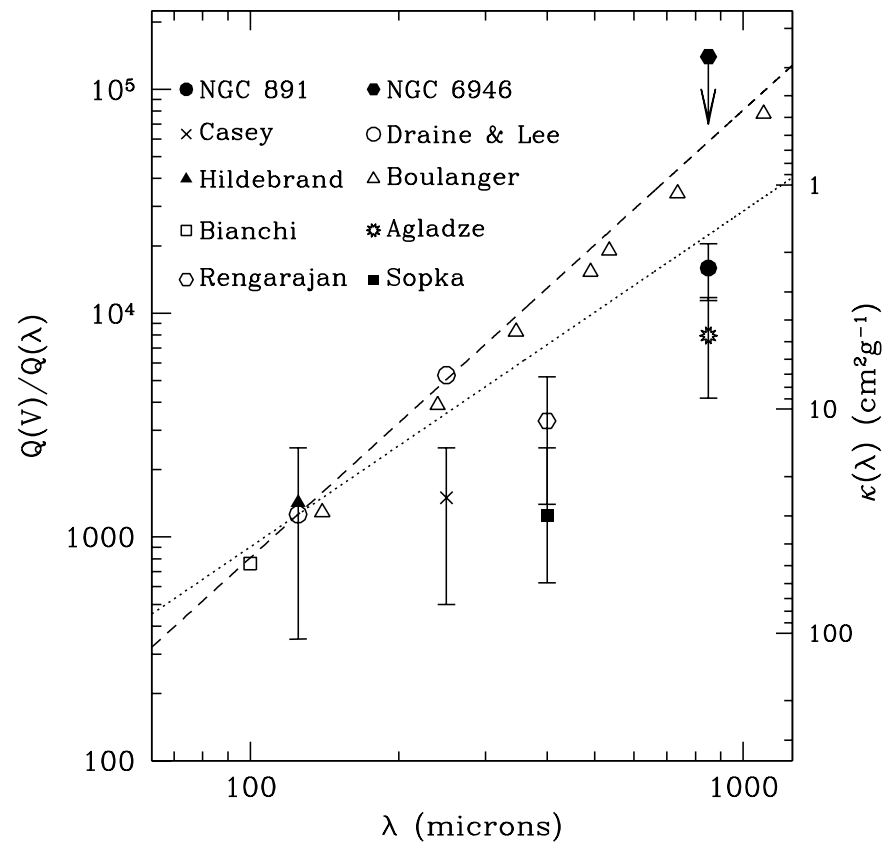

Fig. 7. The emissivity of dust grains in the FIR/submm waveband. Measurements are plotted for Galactic reflection nebulae (Hildebrand 1983; Casey 1991), deeply-embedded FIR sources (Rengarajan 1984), dust envelopes of evolved stars (Sopka et al. 1985), the edge-on spiral NGC 891 (Alton et al. 2000) and NGC 6946 (upper limit, corresponding to our lower limit on submillimeter emissivity given in this paper). Agladze et al. (1994) refers to laboratory experiments conducted on silicate particulates. Draine \& Lee (1984) corresponds to a model for diffuse Galactic dust partly based on empirical data. We also show values derived from COBE and IRAS data based on emission from high-latitude Galactic dust (Boulanger et al. 1996 and Bianchi et al. 1999 respectively). The dotted and dashed lines denote, respectively, $Q(\lambda) \propto \lambda^{-1.5}$ and $Q(\lambda) \propto \lambda^{-2.0}$ and are arbitrarily positioned to pass through the Draine \& Lee $125 \mu \mathrm{m}$ point. The right hand axis shows the equivalent mass absorption coefficient $(\kappa)$ assuming a radius and material density of $0.1 \mu \mathrm{m}$ and $3 \mathrm{~g} \mathrm{~cm}^{-3}$, respectively, for the dust grains and $Q(V)=1.5$.

We should remind here that the molecular gas is dominating in NGC 6946, and its amount is estimated from the $\mathrm{CO}$ emission, which is itself dependent on metallicity. In NGC 891 for example, both CO emission and submillimeter dust emission have the same dependence with radius, suggesting that the $\mathrm{CO}$ emission falls also proportionally to the metallicity (Guélin et al. 1993).

A dashed line in Fig. 8 represents the gas-phase metal abundance as measured from emission-line spectra (VilaCostas \& Edmunds 1992). The vertical axes in the plot have been aligned so that solar metallicity corresponds to a gas-to-dust ratio of 150 . The proximity of our gas-todust estimates to the dashed line is probably fortuitous given the factor 3-4 uncertainty in deriving the former ( $X$ factor, $\mathrm{CO}(2-1) / \mathrm{CO}(1-0)$ etc.).

Nearly no radial variation of the gas-to-dust ratio is observed. This means that the CO tracer is also dependent on metallicity, in a similar way than the dust tracer.

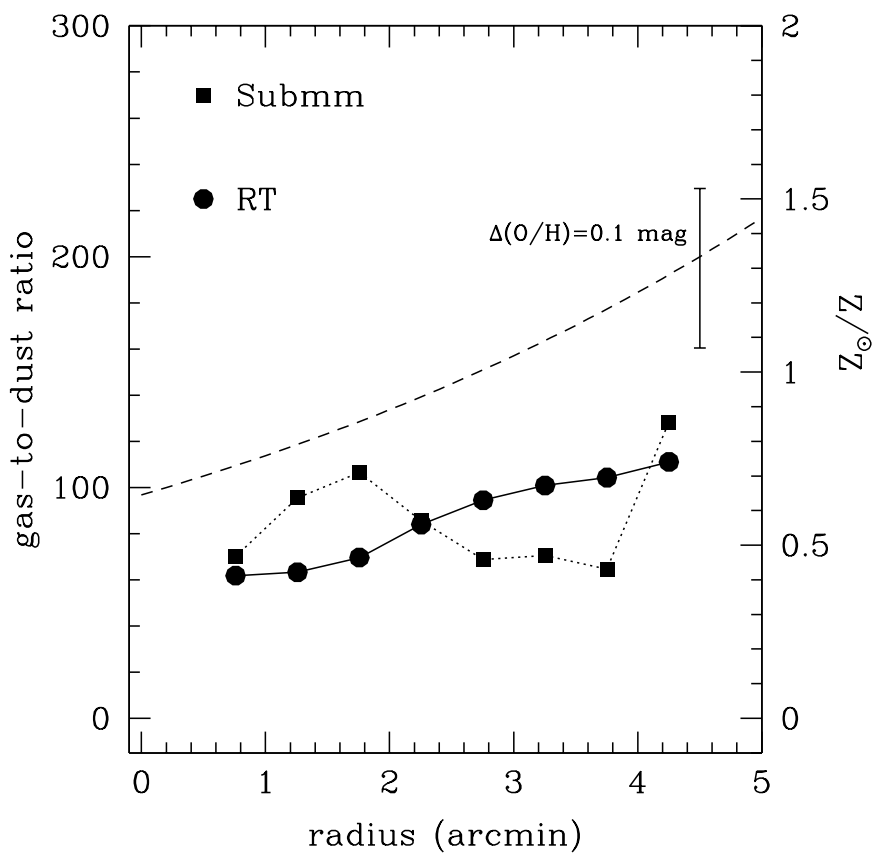

Fig. 8. The radial behaviour in gas-to-dust ratio (left axis) with the dust mass derived from ; (i) RT modelling (solid line) and (ii) $850 \mu \mathrm{m}$ emission (dotted line). The dashed line shows the gas-phase metallicity in NGC 6946 and its associated error (right axis, plotted as $\frac{Z_{\odot}}{Z}$ ). The vertical axes have been aligned so that a gas-to-dust ratio of 150 coincides with solar metallicity. A vertical error-bar shows the probable systematic error in the metallicity measurement (Vila-Costas \& Edmunds 1992).

\section{Dust and gas within arm and interarm regions}

The arm-interarm contrast in dust and gas is difficult to determine because of limited spatial resolution - at $60 \mu \mathrm{m}$, an IRAS beam of $\sim 1^{\prime}$ only begins to resolve spiral disks that are closer than $\sim 5 \mathrm{Mpc}$. SCUBA, with a beam of $16^{\prime \prime}$ at $850 \mu \mathrm{m}$, can probe spiral arms separated by $1^{\prime}$ (such as those in NGC 6946). However, the problem becomes one of sensitivity, with face-on disks possessing surface brightnesses of only a few mJy.

Although our $21 \mathrm{~cm} \mathrm{HI}$ observations are somewhat too coarse for the task (25" FWHM), we have attempted a decomposition of the gas and dust associated with the spiral structure in NGC 6946, concentrating on the NE where the $850 \mu \mathrm{m}$ emission is brightest. A series of 8 crosssections across the arm-interarm interface were produced, sampling the opacity as well as the submm, CO and $21 \mathrm{~cm}$ HI emission. The transects were then stacked, assuming an average arm separation of $60^{\prime \prime}(\lambda)$ and stretching or compressing to take into account the variation of $\lambda$ with galactocentric radius.

Figure 9 depicts the mean profiles generated in this way. The vertical dashed lines define arm and interarm regions on the basis of the $K$-band profile (believed to trace the underlying stellar mass density). All the tracers of dust and gas plotted in Fig. 9 show some modulation with the spiral density wave except perhaps the optical 

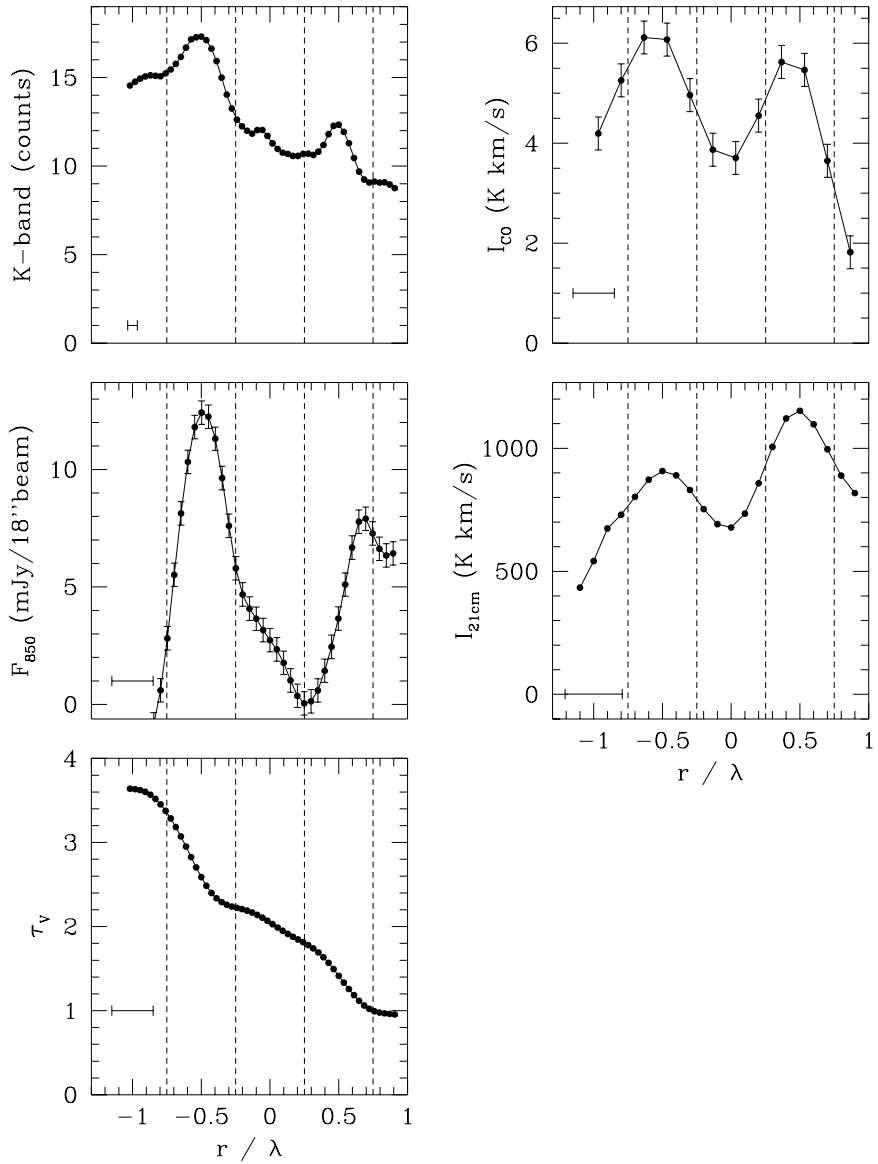

Fig. 9. Profiles of gas and dust across the arm and interarm regions of NGC 6946. In all cases the horizontal axis denotes the distance from the interarm centroid $(r)$ in terms of the mean arm-to-arm separation $(\lambda)$. Dashed vertical lines delineate both the arm regions $(r=-0.75 \lambda$ to $r=-0.25 \lambda$ and $r=+0.25 \lambda$ to $r=+0.75 \lambda)$ and the interarm region $(r=-0.25 \lambda$ to $r=+0.25 \lambda$ ) as defined on the basis of the $K$-band image (topleft). Profiles are shown for $850 \mu \mathrm{m}$ surface brightness $\left(F_{850}\right)$, visual optical depth $\left(\tau_{\mathrm{V}}\right),{ }^{12} \mathrm{CO}(2-1)$ intensity $\left(I_{\mathrm{CO}}\right)$ and intensity in the $21 \mathrm{~cm}$ HI line $\left(I_{21} \mathrm{~cm}\right)$. Errorbars for these quantities are only shown when the uncertainties are much larger than the plotted markers. The spatial resolution of the data is represented by a horizontal error-bar at the bottom-left of each panel.

depth derived from RT modelling. The lack of variation here probably stems from the assumption that an identical intrinsic $B-K$ colour exists for the underlying stellar population in both arm and interarm regions. In reality, the arms will tend to be inherently bluer on average, due to the presence of young stars, leading us underestimate the arm opacity with respect to the interarm value. The two forms of neutral hydrogen seem complementary in the sense that a reduced presence of $\mathrm{HI}$ along one arm is balanced by an enhanced level of $\mathrm{H}_{2}$ and vice versa.

Table 2 contains estimates of the optical depth and gas column density based on the profiles presented in Fig. 9. The visual opacity is calculated variously from: radiative transfer (row 3); $850 \mu \mathrm{m}$ surface brightness using Eq. (5) (row 13); and the gas column density $\mathrm{N}\left(\mathrm{HI}+\mathrm{H}_{2}\right)$ (row 12),
Table 2. Properties of the arm and interarm regions in NGC 6946. Measurements are shown for the following: $K$-band light (row 1), $850 \mu \mathrm{m}$ surface brightness (row 2), visual optical depth (row 3), ${ }^{12} \mathrm{CO}(2-1)$ intensity (row 4), $\mathrm{H}_{2}$ column density (row 5), intensity in the $21 \mathrm{~cm} \mathrm{HI} \mathrm{line} \mathrm{(row} \mathrm{6),} \mathrm{HI} \mathrm{column}$ density (row 7), total hydrogen column density (row 8), ratio of atomic to molecular column density (row 9), visual optical depth inferred from $\mathrm{H}_{2}$ (row 10), visual optical depth inferred from $\mathrm{HI}$ (row 11), visual optical depth inferred from $\mathrm{H}_{2}+\mathrm{HI}$ (row 12) and visual optical depth inferred from the $850 \mu \mathrm{m}$ surface brightness (row 13). The $\mathrm{H}_{2}$ column density assumes $X=1.5 \times 10^{20} \mathrm{~cm}^{-2} \mathrm{~K} \mathrm{~km} \mathrm{~s}^{-1}$ and 0.4 for the ratio of $\mathrm{CO}(2-1)$ to $\mathrm{CO}(1-0)$ intensity. For inferences of visual optical depth see text. Due to the cancellation of systematic errors, uncertainties in the interarm-to-arm ratios are sometimes proportionally smaller than those pertaining to the arm or interarm region alone. Note that none of the quantities appearing in the table have been corrected to face-on values.

\begin{tabular}{lllll}
\hline \hline Quantity & Units & $\begin{array}{l}\text { Arm } \\
\text { average }\end{array}$ & $\begin{array}{l}\text { Interarm } \\
\text { average }\end{array}$ & $\begin{array}{l}\text { Interarm } \\
\text { / Arm }\end{array}$ \\
\hline$K$-band & counts & $13.4 \pm 1.2$ & $11.4 \pm 1.2$ & $0.86 \pm .02$ \\
$F_{850}$ & mJy $/ 18^{\prime \prime}$ & $6.4 \pm 1.5$ & $2.7 \pm 1.5$ & $0.42 \pm 0.3$ \\
$\tau_{\mathrm{V}}$ & & $\leq 2.05$ & $\leq 2.04$ & $\leq 1.0$ \\
$I_{\mathrm{CO}}$ & $\mathrm{K} \mathrm{km} \mathrm{s}^{-1}$ & $5.32 \pm 0.4$ & $4.10 \pm 0.4$ & $0.77 \pm .07$ \\
$N\left(\mathrm{H}_{2}\right)$ & $10^{21} \mathrm{~cm}^{-2}$ & $2.00 \pm 0.1$ & $1.54 \pm 0.1$ & as above \\
$I_{21} \mathrm{~cm}$ & $\mathrm{~K} \mathrm{~km} \mathrm{~s}^{-1}$ & $941 \pm 58$ & $787 \pm 58$ & $0.84 \pm .05$ \\
$N(\mathrm{HI})$ & $10^{21} \mathrm{~cm}^{-2}$ & $1.79 \pm 0.1$ & $1.50 \pm 0.1$ & as above \\
$N\left(\mathrm{H}_{2}+\mathrm{HI}\right)$ & $10^{21} \mathrm{~cm}^{-2}$ & $3.8 \pm 0.2$ & $3.0 \pm 0.2$ & $0.78 \pm .07$ \\
$N\left(\mathrm{H}_{2}\right)$ & & $1.12 \pm 0.1$ & $1.03 \pm 0.1$ & \\
$\tau_{\mathrm{V}}(\mathrm{HI})$ & & $0.96 \pm .08$ & $0.74 \pm .08$ & \\
$\tau_{\mathrm{V}}(\mathrm{HI})$ & & $0.86 \pm .04$ & $0.72 \pm .04$ & \\
$\tau_{\mathrm{V}}\left(\mathrm{H}_{2}+\mathrm{HI}\right)$ & & $1.8 \pm 0.1$ & $1.5 \pm 0.1$ & \\
$\tau_{\mathrm{V}}\left(F_{850}\right)$ & & $3.7 \pm 0.9$ & $1.5 \pm 0.9$ & \\
\hline
\end{tabular}

the last of these assuming $\tau_{\mathrm{B}}-\tau_{\mathrm{V}}=\frac{N\left(\mathrm{HI}+\mathrm{H}_{2}\right)}{6.3 \times 10^{21} \text { atoms } / \mathrm{cm}^{2}}$ as observed in the solar neighborhood (Bohlin et al. 1978). Our estimate of $\tau_{\mathrm{V}}$ based on RT modelling is expected to be an overestimate in the interarm region. Surprisingly, however, the gas column in such interstitial regions suggests equally high values of $\tau_{\mathrm{V}}$. The submm observations favour quite a high interarm opacity as well $(1.5 \pm 0.9)$ but the combination of low signal-to-noise and difficulties in relating grain thermal emission to dust column density make the uncertainties here considerable. Attempts to determine interarm opacity by analysing the colour, surface brightness or number of background galaxies visible through a foreground spiral disk have generally led to values of less than unity for the face-on optical depth (White \& Keel 1992; White et al. 1996; Berlind et al. 1997; Gonzalez et al. 1998).

Although the definition of arms and interarms regions is subjective, we have attempted to compute the gas and dust mass in both regions. We adopt 1.5 and 3.7, respectively, for the optical depth of the interarm and arm region at a radius of $2.3^{\prime}$ (Table 2 ). In addition, we assume a radial scale-length of $98^{\prime \prime}$ for both arm and interstitial environments (Sect. 4). We suppose that interarm regions 
occupy only $50 \%$ of the disk at $R=0.5 \times R_{25}$, increasing linearly with $R$ from $0 \%$ at the nucleus to $100 \%$ at the $R_{25}$ radius. Integrating Eq. (3) suggests that at least $15 \%$ of galactic dust is located between the spiral arms. A similar calculation for $\mathrm{HI}+\mathrm{H}_{2}$ (row 8, Table 2) indicates that at least $68 \%$ of the neutral gas in NGC 6946 can be considered as lying outside the spiral arms.

\section{Summary}

We have analysed $850 \mu \mathrm{m}$ SCUBA images of the nearby, (almost) face-on spiral galaxy NGC 6946. The submillimeter brightness level is low over most of the disk (often a few mJy at $850 \mu \mathrm{m}$ ) and, therefore, care has to be exercised in the subtraction of sky emission. Our main results can be summarised as follows:

1. Comparing the $850 \mu \mathrm{m}$ flux with ${ }^{12} \mathrm{CO}(2-1)$ and $21 \mathrm{~cm}$ $\mathrm{HI}$ line intensity indicates a good correlation between dust thermal emission and CO emission. No correlation was detected between submm emission and atomic gas emission.

2. We place an upper limit on the visual optical depth in NGC $6946\left(\tau_{\mathrm{V}}\right)$ by means of a $B-K$ colour map of the disk. We assume that the $B-K$ colour at a radius $0.9 R_{25}$ corresponds to the underlying intrinsic colour of the stellar disk, in both arm and interarm regions, and that the observed increase in $B-K$ with diminishing radius is attributable solely to extinction by dust. With these conditions we carry out a Monte Carlo radiative transfer simulation of the disk, inferring visual optical depth from $B-K$ colour.

3. The resultant map in $\tau_{\mathrm{V}}$ relates fairly well to the distribution of neutral gas $\left(\mathrm{HI}+\mathrm{H}_{2}\right)$. There is no significant radial variation of the derived gas-to-dust ratio, which is easy to understand, given that the estimated gas content is dominated by the molecular gas, therefore is estimated through the CO emission tracer. Since the $\mathrm{CO}$ emission is also dependent on metallicity, it is not possible to derive the true gas-to-dust ratio. This will await a large-scale mapping of other tracers, such as the pure rotational lines of molecular hydrogen (e.g. Valentijn \& van der Werf 1999).

4. Radial profiles of our optical depth map and SCUBA image indicate an association between $\tau_{\mathrm{V}}$ and (diffuse) submm emission, $F_{850}$. A linear regression yields $\frac{\tau_{\mathrm{V}}}{F_{850}}=573 \pm 110$ and, by corollary, a $850 \mu \mathrm{m}$ emissivity which is no more than 3 times lower than the COBE value for Galactic grains (Boulanger et al. 1996). The emissivity is also 9 times lower than in the edge-on galaxy NGC 891. This is however a lower limit, given the uncertainties on the visual opacity, and the possibility of underestimating the large-scale submm emission. The latter could be estimated in the future by more sensitive and more extended submm arrays.

5. A decomposition of spiral structure in NGC 6946 suggests an interarm optical depth of between 1 and 2 at a mean radius of $0.4 R_{25}$. These surprisingly high val- ues represent $40-80 \%$ of the visual opacity along the arms. Furthermore, by a similar analysis, about twothirds of the neutral gas could be considered as lying outside the spiral arms.

Acknowledgements. It is a pleasure to thank Gerald MoriartySchieven and Tim Jenness for their support during observations and data reduction. We are grateful to Steve Eales and Loretta Dunne for stimulating discussions on grain opacity and related matters, and to the referee for his careful reading and positive criticism.

\section{References}

Agladze, N., Sievers, A., Jones, S., Burlitch, J., \& Beckwith, S. 1994, Nature, 372, 243

Alton, P. B., Trewhella, M., \& Davies, J. 1998a, A\&A, 335, 807

Alton, P., Bianchi, S., Rand, R., et al. 1998b, ApJ, 507, L125

Alton, P., Xilouris, E., Bianchi, S., Davies, J., \& Kylafis, N. 2000, A\&A, 356, 795

Alton, P. B., Lequeux, J., \& Bianchi, S. 2001, A\&A, 366, 451

Beck, R., \& Hoernes, P. 1996, Nature, 379, 47

Beckman, J., Peletier, R., Knapen, J., Corradi, R., \& Gentet, L. 1996, ApJ, 467, 175

Berlind, A., Quillen, A., Pogge, R., \& Sellgren, K. 1997, AJ, 114, 107

Bianchi, S., Ferrara, A., \& Giovanardi, C. 1996, ApJ, 465, 127

Bianchi, S. 1999, Ph.D. Thesis, University of Cardiff

Bianchi, S., Davies, J., \& Alton, P. 1999, A\&A, 344, L1

Bianchi, S., Davies, J., \& Alton, P. 2000a, A\&A, 359, 65

Bianchi, S., Davies, J., Alton, P., Gerin, M., \& Casoli, F. 2000b, A\&A, 353, L13

Bianchi, S., Ferrara, A., Davies, J., \& Alton, P. 2000c, MNRAS, 311,601

Block, D., Witt, A., Grosbol, P., Stockton, A., \& Moneti, A. 1994, A\&A, 288, 383

Bohlin, R., Savage, B., \& Drake, J. 1978, ApJ, 224, 132

Boulanger, F., \& Viallefond, F. 1992, A\&A, 266, 37

Boulanger, F., Abergel, A., Bernard, J. P., et al. 1996, A\&A, 312,256

Burton, W. B., Braun, R., Walterbos, R. A. M., \& Hoopes, C. G. 1999, AJ, 117, 194

Casey, S. 1991, ApJ, 371, 183

Casoli, F., Clausset, F., Combes, F., Viallefond, F., \& Boulanger, F. 1990, A\&A, 233, 357

Corradi, R., Beckman, J., \& Simonneau, E. 1996, MNRAS, 282,1005

de Jong, R. 1996, A\&A, 313, 377

de Vaucouleurs, G., de Vaucouleurs, A., Corwin, H., et al. 1991, Third Reference Catalogue of Bright Galaxies (New York, Springer)

Draine, B., \& Lee, H. 1984, ApJ, 285, 89

Dunne, L., Eales, S., Edmunds, M., et al. 2000, MNRAS, 315, 115

Dumke, M., Braine, J., Krause, M., et al. 1997, A\&A, 325, 124

Dumke, M., Nieten, Ch., Thuma, G., Wielebinski, R., \& Walsh, W. 2001, A\&A, 373, 853

Edmunds, M., \& Eales, S. 1998, MNRAS, 299, L29

Emerson, D. 1995, in Astron. Soc. Pac. Conf. Ser., 75

Engargiola, G. 1991, ApJS, 76, 875 
Fall, M., \& Pei, Y. 1993, ApJ, 402, 479

Frick, P., Beck, R., Berkhuijsen, E. M., \& Patrickeyev, I. 2001, MNRAS, 327, 1145

Genzel, R., \& Cesarsky, C. J. 2000, ARA\&A, 38, 761

Gonzalez, R., Allen, R., Dirsch, B., et al. 1998, ApJ, 506, 152

Guélin, M., Zylka, R., Mezger, P., et al. 1993, A\&A, 279, L37

Guélin, M., Zylka, R., Mezger, P., Haslam, C., \& Kreysa, E. 1995, A\&A, 298, L29

Heisler, J., \& Ostriker J. 1988, ApJ, 332, 543

Hildebrand 1983, QJRAS, 24, 267

Holland, W., Robson, E., Gear, W., et al. 1999, MNRAS, 303, 659

Hughes, D., Robson, E., Dunlop, J., \& Gear, W. 1993, MNRAS, 263, 607

Israel, F., van der Werf, P., \& Tilanus, R. 1999, A\&A, 344, L83

Jenness, T. 1997, Starlink User Note 216.1

Jenness, T., Lightfoot, J., \& Holland, W. 1998, Proc. SPIE, 3357,548

Knapen, J., \& Beckman, J. 1996, MNRAS, 283, 251

Maloney 1990, in Interstellar Medium of Galaxies, ed. H. Thronson, \& J. Shull

Masci, F., \& Webster, R. 1999, MNRAS, 305, 937

Masi, S., Aquilini, E., Boscaleri, A., et al. 1995, ApJ, 452, 253

Mathis, J., Mezger, P., \& Panagia, N. 1983, A\&A, 128, 212

Mauersberger, R., Henkel, C., Walsh, W., \& Schulz, A. 1999, A\&A, 341, 256

Neininger, N., Guélin, M., Garcia-Burillo, S., et al. 1996, A\&A, 310,725

Nieten, Ch., Dumke, M., Beck, R., \& Wielebinski, R. 1999, A\&A, 347, L5
Peletier, R., Valentijn, E., Moorwood, A., et al. 1995, A\&A, 300, L1

Pierce-Price, D., Richer, J., Greaves, J., et al. 2000, ApJ, 545, L121

Rand, R., Lord, S., \& Higdon, J. 1999, ApJ, 513, 720

Reach, W., Dwek, E., Fixsen, D., et al. 1995, ApJ, 451, 188

Rengarajan, T. 1984, A\&A, 140, 213

Sauty, S., Gerin, M., \& Casoli, F. 1998, A\&A, 339, 19

Seigar, M., \& James, P. 1998, MNRAS, 299, 685

Siebenmorgen, R., Kruegel, E., \& Chini, R. 1999, A\&A, 351, 495

Soifer, B., Sanders, D., Madore, B., et al. 1987, ApJ, 320, 238

Sopka, R., Hildebrand, R., Jaffe, D., et al. 1985, ApJ, 294, 242

Tacconi, L., \& Young, J. 1986, ApJ, 308, 600

Tacconi, L., \& Young, J. 1990, ApJ, 352, 595

Trewhella, M. 1997, Ph.D. Thesis, University of Cardiff

Trewhella, M. 1998, MNRAS, 297, 807

Valentijn, E. A., \& van der Werf, P. 1999, ApJ, 522, L29

Vila-Costas, M., \& Edmunds, M. 1992, MNRAS, 259, 121

Weliachew, L., Casoli, F., \& Combes, F. 1988, A\&A, 199, 29

White, R., \& Keel, W. 1992, Nature, 359, 129

White, R., Keel, W., \& Conselice, C. 1996, in New Extragalactic Perspectives in the New South Africa, ed. D. Block, \& M. Greenberg (Kluwer)

Whittet, D. 1992, Dust in the Galactic Environment (IOP Publishing)

Williams, D., \& Taylor, S. 1996, QJRAS, 37, 565

Witt, A., \& Gordon, K. 2000, ApJ, 528, 799

Xilouris, E., Kylafis, N., Papamastorakis, J., Paleologou, E., \& Haerendel, G. 1997, A\&A, 325, 135 